\title{
Sofosbuvir/Velpatasvir for Hepatitis C Virus Infection: Real-World Effectiveness and Safety from a Nationwide Registry in Taiwan
}

\author{
Pin-Nan Cheng $\cdot$ Lein-Ray Mo • Chun-Ting Chen • Chi-Yi Chen • Chung-Feng Huang • Hsing-Tao Kuo • \\ Ching-Chu Lo · Kuo-Chih Tseng · Yi-Hsiang Huang · Chi-Ming Tai • Cheng-Yuan Peng · Ming-Jong Bair • \\ Chien-Hung Chen · Ming-Lun Yeh · Chih-Lang Lin · Chun-Yen Lin · Pei-Lun Lee · Lee-Won Chong • \\ Chao-Hung Hung · Te Sheng Chang · Jee-Fu Huang • Chi-Chieh Yang · Jui-Ting Hu • Chih-Wen Lin • \\ Chia-Chi Wang · Wei-Wen Su • Tsai-Yuan Hsieh • Chih-Lin Lin • Wei-Lun Tsai • Tzong-Hsi Lee • \\ Guei-Ying Chen · Szu-Jen Wang • Chun-Chao Chang · Sheng-Shun Yang • Wen-Chih Wu • Chia-Sheng Huang • \\ Kwok-Hsiung Chou · Chien-Neng Kao · Pei-Chien Tsai · Chen-Hua Liu • Mei-Hsuan Lee $\cdot$ Chien-Yu Cheng • \\ Ming-Chang Tsai · Chun-Jen Liu · Chia-Yen Dai · Han-Chieh Lin · Jia-Horng Kao • Wan-Long Chuang • \\ Ming-Lung Yu (D) on behalf of TACR investigators
}

Received: October 23, 2021 / Accepted: November 29, 2021 / Published online: December 28, 2021 (c) The Author(s) 2021

\section{ABSTRACT}

Introduction: Pangenotypic direct-acting antivirals are expected to cure hepatitis $C$ virus $(\mathrm{HCV})$ in more than $95 \%$ of treated patients.

Supplementary Information The online version contains supplementary material available at https:// doi.org/10.1007/s40121-021-00576-7.

P.-N. Cheng

Division of Gastroenterology and Hepatology, Department of Internal Medicine, National Cheng Kung University Hospital, College of Medicine, National Cheng Kung University, Tainan, Taiwan

\section{L.-R. Mo}

Division of Gastroenterology, Tainan Municipal Hospital, Tainan, Taiwan

C.-T. Chen · K.-H. Chou

Division of Gastroenterology, Department of Internal Medicine, Tri-Service General Hospital Penghu Branch, National Defense Medical Center, Taipei, Taiwan

C.-T. Chen · T.-Y. Hsieh · K.-H. Chou Division of Gastroenterology, Department of Internal Medicine, Tri-Service General Hospital, National Defense Medical Center, Taipei, Taiwan
However, data on the effectiveness and safety of sofosbuvir/velpatasvir (SOF/VEL) in Taiwan are limited. This study aims to characterize the patient population in the nationwide Taiwan Association for the Study of the Liver (TASL) HCV Registry and evaluate treatment outcome in Taiwanese patients receiving SOF/VEL.

Methods: This study was a retrospectiveprospective, observational, multicenter, real-

C.-Y. Chen

Division of Gastroenterology and Hepatology, Department of Medicine, Ditmanson Medical Foundation Chiayi Christian Hospital, Chiayi, Taiwan

C.-F. Huang · W.-C. Wu · P.-C. Tsai · C.-Y. Dai · W.-L. Chuang · M.-L. Yu ( $\varangle)$

Hepatobiliary Division, Department of Internal Medicine and Hepatitis Center, Kaohsiung Medical University Hospital, Kaohsiung Medical University, Kaohsiung, Taiwan

e-mail: fish6069@gmail.com

C.-F. Huang · M.-L. Yeh · J.-F. Huang · C.-Y. Dai ·

W.-L. Chuang · M.-L. Yu

School of Medicine and Hepatitis Research Center, College of Medicine, and Center for Cancer Research and Center for Liquid Biopsy and Cohort Research, Kaohsiung Medical University, Kaohsiung, Taiwan 
world analysis. Adults with chronic hepatitis C were treated with SOF/VEL 400/100 $\mathrm{mg} \pm$ ribavirin for 12 weeks. The primary outcome was sustained virologic response 12 weeks after end of therapy (SVR12). Factors associated with not achieving SVR12 were evaluated using logistic regression and covariate analysis. Safety was also assessed.

Results: In total, 3480 patients were included: $86.8 \%$ genotype $1 / 2,2.8 \%$ genotype $3,0.1 \%$ genotype $4 / 5,9.6 \%$ genotype 6 ; unclassified, $0.8 \% ; 12.2 \%$ compensated cirrhosis; $3.3 \%$ decompensated cirrhosis; and $15.8 \%$ chronic kidney disease. Overall SVR12 rate was $99.4 \%$ (genotype 1, 99.5\%; genotype 2, 99.4\%; genotype 3, 96.9\%; genotype 4, 100\%; genotype 6 , 99.7\%). SVR12 rates among patients with compensated cirrhosis, decompensated cirrhosis, and chronic kidney disease stages 4-5 were $99.5 \%, 100 \%$, and $100 \%$, respectively. There were 21 patients $(0.6 \%)$ who did not achieve SVR12. Factors associated with failure were treatment adherence below 60\%, high viral

\section{H.-T. Kuo}

Division of Gastroenterology and Hepatology, Department of Internal Medicine, Chi Mei Medical Center, Yongkang District, Tainan, Taiwan

C.-C. Lo

Division of Gastroenterology, Department of Internal Medicine, St. Martin De Porres Hospital, Chiayi, Taiwan

\section{K.-C. Tseng}

Department of Internal Medicine, Dalin Tzu Chi Hospital, Buddhist Tzu Chi Medical Foundation, Chiayi, Taiwan

\section{K.-C. Tseng}

School of Medicine, Tzuchi University, Hualien, Taiwan

Y.-H. Huang · H.-C. Lin

Division of Gastroenterology and Hepatology, Department of Medicine, Taipei Veterans General Hospital, Taipei, Taiwan

\section{Y.-H. Huang · H.-C. Lin ( $\square)$}

Institute of Clinical Medicine, School of Medicine, National Yang Ming Chiao Tung University, Taipei, Taiwan

e-mail: hclin@vghtpe.gov.tw

\section{C.-M. Tai}

Department of Internal Medicine, E-Da Hospital, Kaohsiung, Taiwan load, and genotype 3 ( $p<0.001, p=0.028$, and $p=0.001$, respectively). Adverse events occurred in $10 \%$ of patients; $0.6 \%$ were serious and one was related to treatment. Treatment discontinuation occurred in $0.3 \%$ of patients; none were treatment related. The estimated glomerular filtration rate remained stable throughout treatment and follow-up, regardless of baseline values and cirrhosis status. Conclusion: SOF/VEL was highly effective and well tolerated in Taiwanese patients, irrespective of viral genotype, liver disease severity, and comorbidities.

Keywords: Hepatitis C; Sofosbuvir; Velpatasvir; Direct-acting antivirals; Real-world; Taiwan

C.-M. Tai

School of Medicine, College of Medicine, I-Shou University, Kaohsiung, Taiwan

C.-Y. Peng

Center for Digestive Medicine, Department of Internal Medicine, China Medical University Hospital, Taichung, Taiwan

\section{C.-Y. Peng}

School of Medicine, China Medical University, Taichung, Taiwan

M.-J. Bair

Division of Gastroenterology, Department of Internal Medicine, Taitung Mackay Memorial Hospital, Taitung, Taiwan

M.-J. Bair

Mackay Medical College, New Taipei City, Taiwan

C.-H. Chen · C.-H. Hung

Division of Hepato-Gastroenterology, Department of Internal Medicine, Kaohsiung Chang Gung Memorial Hospital and Chang Gung University College of Medicine, Taoyuan City, Taiwan

\section{M.-L. Yeh}

Department of Internal Medicine, Kaohsiung Municipal Siaogang Hospital, Kaohsiung Medical University Hospital, Kaohsiung Medical University, Kaohsiung, Taiwan 


\section{Key Summary Points}

\section{Why carry out this study?}

Data on hepatitis C virus (HCV) treatment in certain populations in Taiwan are limited.

Data on HCV treatment with sofosbuvir/ velpatasvir (SOF/VEL) from large-scale real-world settings in Taiwan are limited.

Efficacy and safety of SOF/VEL was analyzed in 3480 patients using a nationwide $\mathrm{HCV}$ database.

\section{What was learned from this study?}

Overall, 99.4\% of patients with $\mathrm{HCV}$ treated with SOF/VEL, with and without ribavirin, achieved cure, regardless of baseline characteristics and patient population.

Treatment with SOF/VEL was well tolerated in those with severe liver disease and comorbidities.

Treatment with SOF/VEL did not worsen kidney function in patients with HCV and chronic kidney disease.

C.-L. Lin

Department of Gastroenterology and Hepatology, Keelung Chang Gung Memorial Hospital, Keelung, Taiwan

C.-Y. Lin

Department of Gastroenterology and Hepatology, Chang Gung Memorial Hospital, Linkou Branch, Taiwan

C.-Y. Lin

Graduate Institute of Biomedical Science, College of Medicine, Chang Gung University, Taoyuan,

Taiwan

P.-L. Lee

Liouying Division of Gastroenterology and Hepatology, Department of Internal Medicine, Chi Mei Medical Center, Tainan, Taiwan

\section{L.-W. Chong}

Division of Hepatology and Gastroenterology, Department of Internal Medicine, Shin Kong Wu Ho-Su Memorial Hospital, Taipei, Taiwan

\section{INTRODUCTION}

Hepatitis C virus (HCV) is a global public health concern, with over 71 million people infected around the world. Chronic $\mathrm{HCV}$ is recognized as a common cause of cirrhosis, hepatocellular carcinoma (HCC), liver transplantation, and liver-related deaths [1]. Since the introduction of direct-acting antivirals (DAAs), the expectation is that $95 \%$ of treated patients will be cured of $\mathrm{HCV}$-defined as a sustained virologic response 12 weeks after the end of therapy (SVR12) [2]. Achieving SVR12 is associated with a decreased risk of liver disease progression and its complications, including liver decompensation, portal hypertension, and death $[3,4]$.

Sofosbuvir/velpatasvir (SOF/VEL), a protease inhibitor (PI)-free 12-week regimen for patients with chronic HCV, has demonstrated SVR12 rates of $95-100 \%$ in over 1100 clinical trial patients with HCV genotypes 1-6 [5-7]. Efficacy in clinical trials has translated into effectiveness in clinical practice, evidenced through multiple real-world studies [8-10]. Clinical trials in an Asian population have shown similar results to those in western parts of the world [11].

Many regions in Taiwan are classified as hyperendemic for $\operatorname{HCV}[12,13]$, with a

\author{
L.-W. Chong \\ School of Medicine, Fu-Jen Catholic University, \\ New Taipei City, Taiwan
}

C.-H. Hung · T. S. Chang

Division of Hepatogastroenterology, Department of Internal Medicine, ChiaYi Chang Gung Memorial Hospital and College of Medicine, Chang Gung University, Taoyuan, Taiwan

\section{J.-F. Huang}

Department of Internal Medicine, Kaohsiung Municipal Ta-Tung Hospital, Kaohsiung Medical University Hospital, Kaohsiung Medical University, Kaohsiung, Taiwan

C.-C. Yang

Department of Gastroenterology, Division of Internal Medicine, Show Chwan Memorial Hospital, Changhua, Taiwan

J.-T. Hu

Liver Center, Cathay General Hospital, Taipei, Taiwan 
prevalence rate of $1.8-5.5 \%$ [14]. Genotypes 1 and 2 are the most frequent in the general population [15] and genotypes 1, 3, and 6 are the most dominant among people who inject drugs (PWID) [16]. The use of SOF/VEL has been approved in Taiwan for HCV-infected patients with compensated liver disease, and with the addition of ribavirin it can be used to treat patients with decompensated cirrhosis [17].

Real-world data of DAA therapy for chronic hepatitis $C$ have been reported extensively in Europe and North America. Although some

C.-W. Lin

Division of Gastroenterology and Hepatology, E-Da Dachang Hospital, and School of Medicine, College of Medicine, I-Shou University, Kaohsiung, Taiwan

C.-C. Wang

Taipei Tzu Chi Hospital, Buddhist Tzu Chi Medical Foundation and School of Medicine, Tzu Chi

University, Hualien, Taiwan

W.-W. Su

Department of Gastroenterology and Hepatology, Changhua Christian Hospital, Changhua, Taiwan

C.-L. Lin

Department of Gastroenterology, Taipei City

Hospital, Renai Branch, Taipei, Taiwan

W.-L. Tsai

Division of Gastroenterology and Hepatology,

Department of Internal Medicine, Kaohsiung

Veterans General Hospital, Kaohsiung, Taiwan

T.-H. Lee

Division of Gastroenterology and Hepatology, Far

Eastern Memorial Hospital, New Taipei, Taiwan

G.-Y. Chen

Penghu Hospital, Ministry of Health and Welfare, Penghu, Taiwan

\section{S.-J. Wang}

Division of Gastroenterology, Department of Internal Medicine, Yuan's General Hospital, Kaohsiung, Taiwan

\section{C.-C. Chang}

Division of Gastroenterology and Hepatology, Department of Internal Medicine, Taipei Medical University Hospital, Taipei, Taiwan

\section{C.-C. Chang}

Division of Gastroenterology and Hepatology, Department of Internal Medicine, School of Medicine, College of Medicine, Taipei Medical University, Taipei, Taiwan real-world evidence has been published recently, there is still a lack of data in some patient populations in Taiwan, particularly those with decompensated cirrhosis.

The Taiwan Association for the Study of the Liver (TASL) HCV Registry (TACR) is a nationwide registry program to manage the database and biobank of patients with HCV who receive DAA therapy in Taiwan. By February 2021, a total of 48 sites, including 22 regional hospitals, 21 medical centers, and 5 primary care clinics,

S.-S. Yang

Division of Gastroenterology and Hepatology,

Department of Internal Medicine, Taichung

Veterans General Hospital, Taichung, Taiwan

C.-S. Huang

Yang-Ming Hospital, Taipei, Taiwan

C.-N. Kao

National Taiwan University Hospital Hsin-Chu Branch, Hsin-Chu, Taiwan

C.-H. Liu · C.-J. Liu · J.-H. Kao

Hepatitis Research Center and Department of Internal Medicine, National Taiwan University Hospital, National Taiwan University, Taipei, Taiwan

M.-H. Lee

Institute of Clinical Medicine, School of Medicine, National Yang Ming Chiao Tung University, Taipei, Taiwan

M.-H. Lee · C.-Y. Cheng

Institute of Public Health, School of Medicine,

National Yang Ming Chiao Tung University, Taipei, Taiwan

C.-Y. Cheng

Department of Infectious Diseases, Taoyuan General Hospital, Ministry of Health and Welfare, Taoyuan, Taiwan

M.-C. Tsai

Division of Gastroenterology and Hepatology, Department of Internal Medicine, Chung Shan Medical University Hospital, Institute of Medicine, School of Medicine, Chung Shan Medical University, Taichung, Taiwan 
were contributing patient data to the registry [18].

The current study aimed to use the TACR database and biobank to characterize the patient population and evaluate the treatment outcomes of patients with chronic HCV receiving SOF/VEL in Taiwan, particularly in patient populations where little or no data are available.

\section{METHODS}

\section{Design}

This study was a retrospective-prospective, observational, multicenter, real-world analysis of patients who received a 12-week fixed dose of SOF/VEL \pm ribavirin from 2017 to February 2021. Data from the TACR database were used, details of which have been published previously [19]. The study was approved by the institutional review board at each study site, conformed to the guidelines of the International Conference on Harmonization for Good Clinical Practice, and was performed in accordance with the Helsinki Declaration of 1964 and its later amendments. This study included a retrospective cohort from which data were retrieved retrospectively and a prospective cohort where data were collected prospectively. Informed consent for the data collected retrospectively was waived by the ethics committee. All patients participating in the prospective cohort provided written informed consent.

\section{Patient Population}

Patients with detectable HCV RNA receiving SOF/VEL 400/100 mg \pm ribavirin, once daily for 12 weeks before the end of February 2021 were eligible for inclusion. Patients included in the current analysis were aged over 20 years, had received at least one dose of medication, and completed post-treatment follow-up with SVR12 data available. Patients were treated according to local standards of care, with comorbidities determined by the treating physician according to local clinical practice. No specific exclusion criteria, including severe renal impairment, were applied to the study population. The study complied with all necessary ethics guidance.

\section{Outcomes}

Effectiveness, defined as HCV RNA negativity 12 weeks after the end of therapy, was assessed in all patients receiving at least one dose of SOF/ VEL with a valid SVR12 status available. The primary outcome was the proportion of patients achieving SVR12. Pill counts were used to estimate treatment adherence. Factors associated with virologic failure were evaluated using logistic regression and covariate analysis. Among patients with decompensated cirrhosis, degree of conserved liver function was categorized using the Child-Turcotte-Pugh (CTP) score before starting therapy and 12 weeks after SOF/VEL therapy. Clinical judgement of the treating physician determined whether any adverse events were related to the study medication.

\section{Statistical Analysis}

Descriptive characteristics were presented as the number $(n)$ and percentage of patients (\%) for categorical variables. Continuous variables were summarized as mean ( \pm SD). The effect of patient characteristics, including injecting drug use, HBV or HIV coinfection, cirrhosis, presence of HCC, renal function, presence of chronic kidney disease (CKD), HCV viral load, HCV genotype, treatment history, drug adherence, treatment duration, and addition of ribavirin were evaluated using logistic regression. Multivariate regression analysis was performed to determine the factors associated with failure to achieve SVR12 by analyzing the covariates with a $p$ value less than 0.1 in the univariate analysis. Analyses were performed using the SPSS 12.0 statistical package. 
Table 1 Baseline demographics

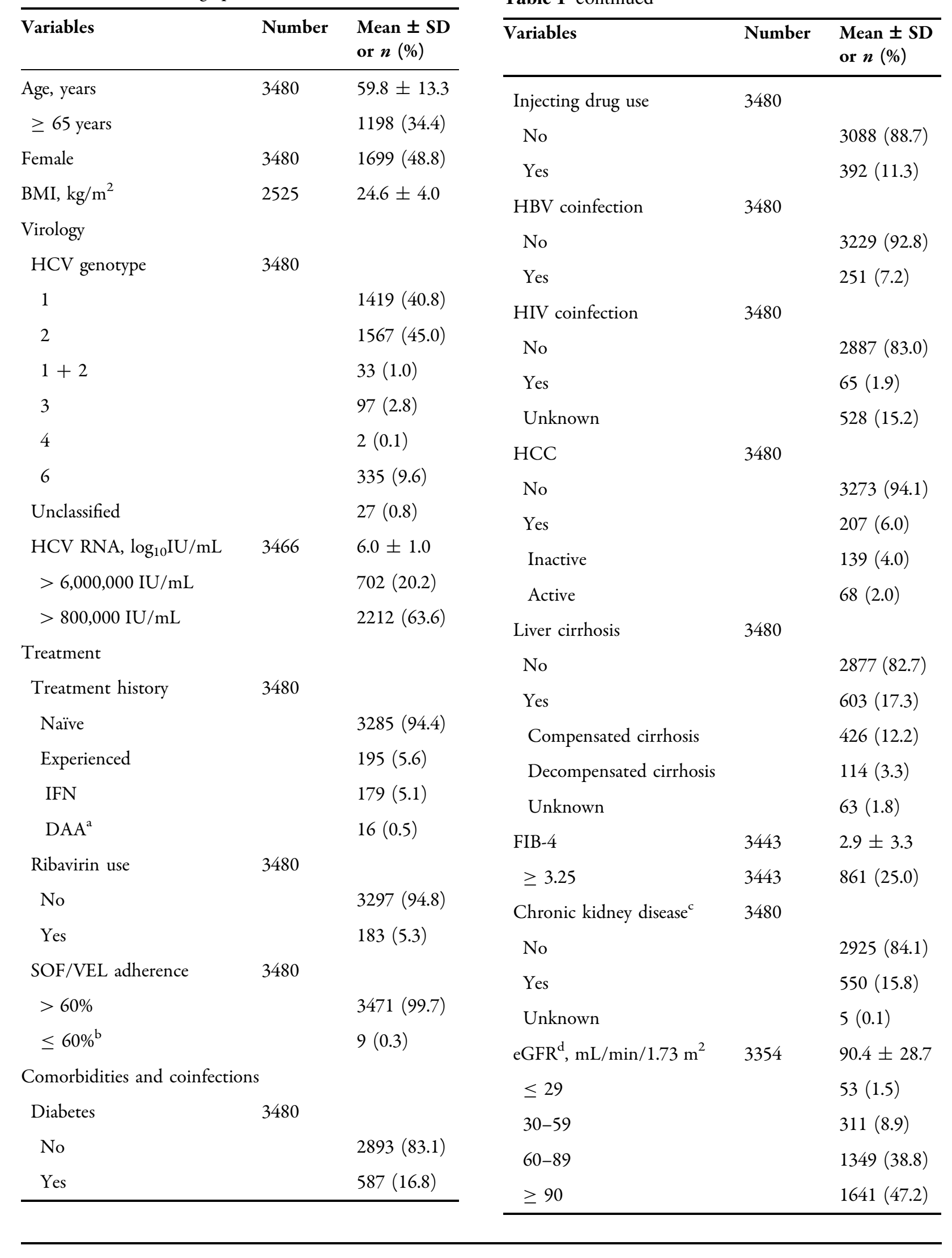

Table 1 continued 
Table 1 continued

\begin{tabular}{lll}
\hline Variables & Number & $\begin{array}{l}\text { Mean } \pm \text { SD } \\
\text { or } \boldsymbol{n}(\%)\end{array}$ \\
\hline $\begin{array}{l}\text { Liver transplantation } \\
\text { Laboratory assessments }\end{array}$ & 3480 & $7(0.2)$ \\
AST, IU/L & 3469 & $56.4 \pm 58.6$ \\
ALT, IU/L & 3472 & $70.9 \pm 96.0$ \\
r-GT, U/L & 1388 & $60.4 \pm 87.6$ \\
Platelet count, $\times 10^{3} / \mu \mathrm{L}$ & 3456 & $193.8 \pm 74.0$ \\
Albumin, g/dL & 3405 & $4.2 \pm 0.5$ \\
Total bilirubin, mg/dL & 3458 & $0.8 \pm 0.6$ \\
Creatinine ${ }^{\mathrm{d}}, \mathrm{mg} / \mathrm{dL}$ & 3354 & $0.9 \pm 0.7$ \\
\hline
\end{tabular}

ALT alanine aminotransferase, $A S T$ aspartate aminotransferase, $B M I$ body mass index, $D A A$ direct-acting antiviral, $e G F R$ estimated glomerular filtration rate, $F I B-4$ fibrosis-4, IFN interferon, HCC hepatocellular carcinoma, $r$ - $G T$ gamma-glutamyl transferase

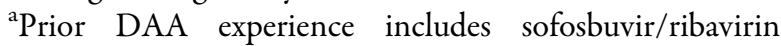
$(n=8) ; \quad$ sofosbuvir/ledipasvir \pm ribavirin $\quad(n=3)$; ombitasvir/paritaprevir/dasabuvir \pm ribavirin $\quad(n=2)$; sofosbuvir/daclatasvir \pm ribavirin $(n=1)$; daclatasvir/ asunaprevir \pm ribavirin $\quad(n=1) ; \quad$ elbasvir/grazoprevir $(n=1)$

${ }^{\mathrm{b}}$ Reasons for adherence of $\leq 60 \%$ : early treatment discontinuation ( $n=4$; acute myocardial infarction $[n=1]$, gastrointestinal upset, nausea, epigastralgia $[n=1]$, dry mouth, dizziness, epigastralgia $[n=1]$, unwillingness $[n=1])$ and treatment interruption $(n=5$; headache $[n=1]$, epigastralgia $[n=1]$, trauma $[n=1]$, hospital admission due to pneumonia $[n=1]$, insomnia $[n=1]$ ) ${ }^{\mathrm{c} D e f i n e d}$ as patients on regular dialysis, or $\mathrm{eGFR} \leq 59 \mathrm{~mL} / \mathrm{min} / 1.73 \mathrm{~m}^{2}$ or $\mathrm{eGFR} \geq 60 \mathrm{~mL} / \mathrm{min} /$ $1.73 \mathrm{~m}^{\overline{2}}$ if combined with proteinuria (dialysis patients: 126 patients; $\quad \mathrm{eGFR} \geq 60 \mathrm{~mL}+$ proteinuria: 147 patients)

${ }^{\mathrm{d}} 126$ dialysis patients were excluded

\section{RESULTS}

\section{Baseline Characteristics}

Overall, 3480 patients with HCV were included in the study, with a mean age of $59.8 \pm 13.3$ years. Baseline characteristics are included in Table 1 and further details are provided in the Supplementary Material (Table S1). Overall, $48.8 \%$ were female, $11.3 \%$ were active injection drug users, and $5.6 \%$ had previous treatment experience. Additionally, over $85 \%$ of patients had genotypes 1 and/or 2, 12.2\% of patients had compensated cirrhosis, and 3.3\% had decompensated cirrhosis $(n=114)$. In terms of coinfection, $7.2 \%$ were coinfected with HBV and $1.9 \%$ were coinfected with HIV. Furthermore, $15.8 \%$ had CKD, $16.8 \%$ had diabetes, $28.2 \%$ had hypertension, and 6\% had HCC ( $2 \%$ active cases). Over the course of treatment, patients attended a mean of $5.6( \pm 0.6$, range 2-9) outpatient visits.

\section{Effectiveness}

Overall, $99.4 \%$ of patients achieved SVR12. When stratified by genotype, SVR12 rates were generally between $96.9 \%$ and 100\% and were largely similar with and without ribavirin (Fig. 1a). SVR12 in patients with compensated cirrhosis was $99.5 \%$. In patients with genotype 3 infection, SVR12 rates were $94.1 \%$ and $100 \%$ in patients with compensated cirrhosis and decompensated cirrhosis, respectively [Supplementary Material (Table S2)].

Further subgroup analyses demonstrated SVR12 rates of between $97.6 \%$ and $99.5 \%$ when stratified by treatment history and presence of cirrhosis (Fig. 1b). Of the patients who were previously treated with interferon-based regimens and DAA-based regimens, $98.3 \%$ and 93.8\% achieved SVR12, respectively. All patients with grade 3-4 total bilirubin, and the majority of patients with grade $2-4$ alanine aminotransferase (ALT) elevations, achieved SVR12.

Overall, adherence was greater than $60 \%$ in $99.7 \%$ of patients and SVR12 rates in this population were at least $99 \%$, irrespective of viral load. In the difficult-to-cure subpopulation, of those infected with genotype 3 with a high viral load (greater than 6,000,000 IU/mL), all achieved SVR12 (Fig. 1c).

The majority of patients were treated with SOF/VEL in accordance with the product label in Taiwan; $99.5 \%$ of these patients achieved 

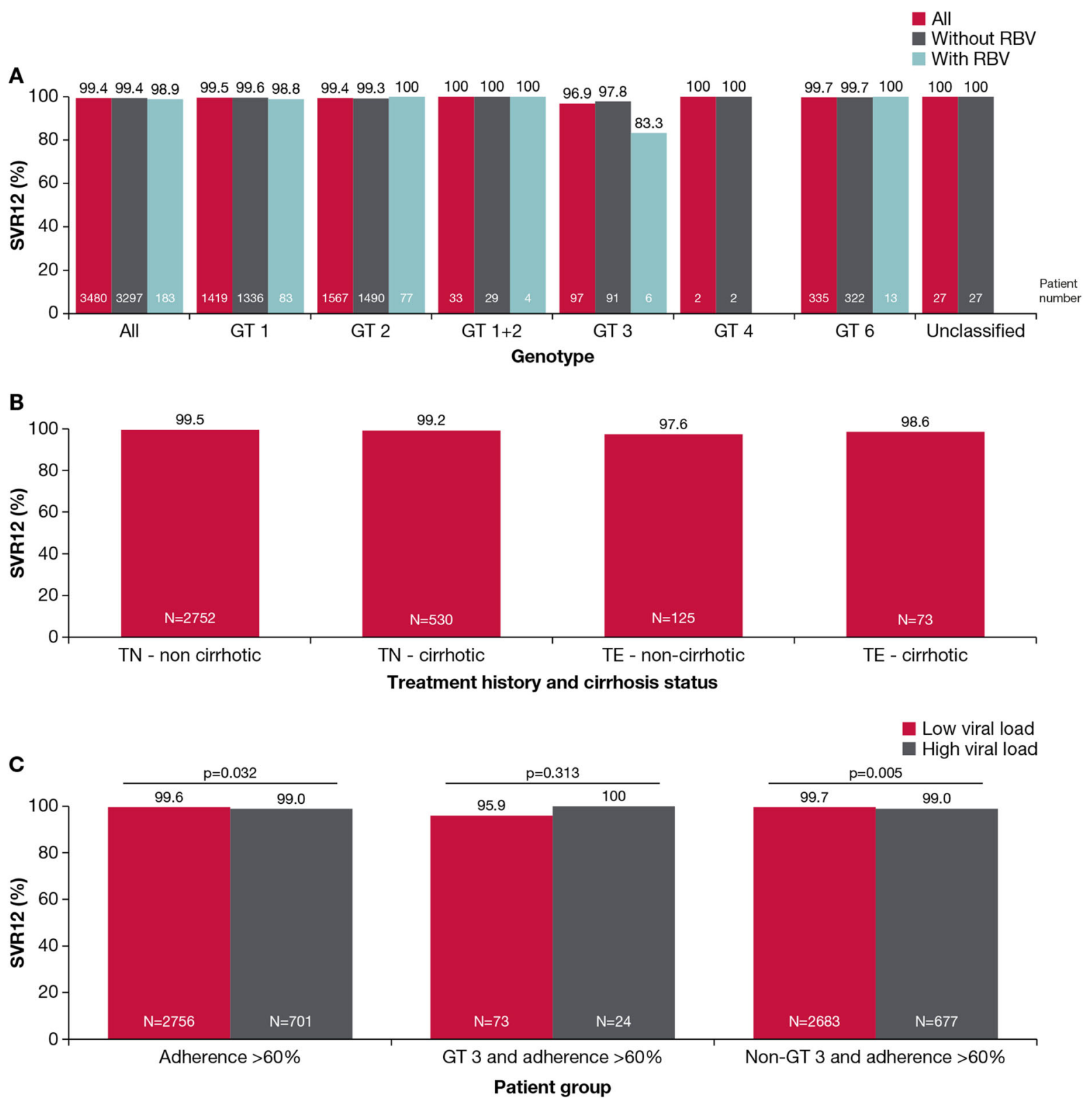

Fig. 1 SVR12 in different patient populations. Percentage of patients achieving SVR12 stratified by a genotype and ribavirin use, $\mathbf{b}$ treatment history and cirrhosis stage, and c genotype, viral load, and adherence. High viral load was

SVR12. Of the 132 patients not treated in accordance with the product label, 97.0\% achieved SVR12 [Supplementary Material (Table S3)].

This study included a large proportion of patients from special populations, including those with $\mathrm{CKD}$ or decompensated liver defined as greater than $6,000,000 \mathrm{IU} / \mathrm{mL}$ [20]. $G T$ genotype, $R B V$ ribavirin, SVR12 sustained virologic response 12 weeks after end of treatment, $T E$ treatmentexperienced, $T N$ treatment-naïve

cirrhosis. All 25 patients with CKD stage 4 or stage 5 [estimated glomerular filtration rate $($ eGFR $)<30 \mathrm{~mL} / \mathrm{min} / 1.73 \mathrm{~m}^{2}$ ] not under dialysis achieved SVR12. Of the 126 patients under maintenance dialysis, 96.8\% attained SVR12.

All 114 patients with decompensated liver cirrhosis at baseline achieved SVR12, of which 
$34 \%$ were treated without ribavirin. Of the patients with decompensated cirrhosis, 85 had CTP scores available at both baseline and at follow-up. Among these 85 patients, $70.6 \%$ had lower CTP scores at end of follow-up versus baseline, $17.6 \%$ had no change, and $11.7 \%$ had higher CTP scores [Supplementary Material (Fig. S1)]. For the remaining 29 patients, end-offollow-up CTP scores were missing.

\section{Factors Associated with Failure to Achieve SVR12}

Overall, 21 patients did not achieve SVR12, with four of these patients failing because of nonvirologic reasons (adherence less than 20\%, $n=3$; and $20-40 \%, n=1$ ). Of the remaining patients, 15 experienced a relapse, and two were non-responders. Detailed baseline characteristics of patients who did not achieve SVR12 are provided in the Supplementary Material (Table S4).

Logistic regression analyses were performed to investigate the impact of different patient characteristics on treatment outcome. Following an adjusted crude odds ratios (OR) analysis, drug adherence below $60 \%$ was the most critical factor associated with the risk of not achieving SVR12 [adjusted OR $=173.37,95 \%$ confidence interval (CI) 27.09-1109.57]. HCV RNA viral load above $6,000,000 \mathrm{IU} / \mathrm{mL}$ and $\mathrm{HCV}$ genotype 3 were found to be associated with the risk of not achieving SVR12 [adjusted OR $=3.39$ (95\% CI 1.13-10.13) and adjusted OR $=11.01$ (95\% CI 2.61-46.37), respectively] [Supplementary Material (Table S5)].

\section{Safety}

Discontinuation of treatment was observed in $0.3 \%$ of the cohort and $33 \%$ of discontinuations were due to adverse events. None of the treatment discontinuations were considered related to treatment with SOF/VEL (Table 2). Adverse events were reported in $10 \%$ of the cohort. The most common adverse events were fatigue, insomnia, pruritus, and headache, with fatigue and headache occurring significantly more frequently in patients receiving ribavirin.
Grade 3-4 total bilirubin and grade 3-4 ALT elevations were observed in $1.2 \%$ of patients and $0.3 \%$ of patients, respectively. Milder increases in total bilirubin were more common in patients receiving ribavirin. None were found to be related to treatment. Serious adverse events were reported in $0.6 \%$ of patients, of which one was related to treatment. The most frequently observed serious adverse events were liver-related $(n=5)$ or infections $(n=6)$.

\section{Impact on Renal Function}

eGFR levels remained stable through end of treatment to end of follow-up, irrespective of cirrhosis status (Fig. 2; Supplementary Material, Table S6), baseline renal function (Supplementary Material, Fig. S2A), or treatment with ribavirin (Supplementary Material, Fig. S2B). A significant improvement in renal function was observed in patients with cirrhosis and eGFR $<90 \mathrm{~mL} / \mathrm{min} / 1.73 \mathrm{~m}^{2}$ from baseline to end of treatment $(p=0.004)$ and baseline to end of follow-up $(p=0.009)$.

\section{DISCUSSION}

This study is the largest real-world study in Asia to evaluate the effectiveness and safety of SOF/ VEL in a diverse group of patients with a broad range of HCV genotypes, comorbidities, and liver disease severity.

Although there have been some real-world analyses on the use of SOF/VEL in Taiwan published recently, there is still a gap in data available. The studies that have been published to date have reported high SVR12 rates (between $98 \%$ and $100 \%)[19,21]$. A study using the TACR database previously investigated the efficacy of SOF/VEL, reporting SVR12 rates of 99\%. However, only 390 patients were included [18]. In comparison, the large-scale TACR study reported here, which includes 3480 patients treated with SOF/VEL in Taiwan, confirms the data from clinical trials and real-world evidence, supporting the use of SOF/VEL in clinical practice across diverse patient groups.

In the current study, high SVR12 rates were achieved across all genotypes, including 
Table 2 Adverse events

\begin{tabular}{|c|c|c|c|c|}
\hline & Total $(n=3480)$ & + Ribavirin $(n=183)$ & - Ribavirin $(n=3297)$ & $P$ value \\
\hline Treatment discontinuation, $n(\%)$ & $10(0.3)$ & $0(0.0)$ & $10(0.3)$ & - \\
\hline Adverse event-related & $3(0.1)$ & $0(0.0)$ & $3(0.1)$ & - \\
\hline Treatment-related & $0(0.0)$ & $0(0.0)$ & $0(0.0)$ & - \\
\hline Any adverse event, $n(\%)$ & $348(10.0)$ & $43(23.5)$ & $305(9.3)$ & $<0.001$ \\
\hline Fatigue & $152(4.4)$ & $23(12.6)$ & $129(3.9)$ & $<0.001$ \\
\hline Insomnia & $49(1.4)$ & $4(2.2)$ & $45(1.4)$ & 0.359 \\
\hline Pruritus & $44(1.3)$ & $3(1.6)$ & $41(1.2)$ & 0.641 \\
\hline Headache & $40(1.2)$ & $5(2.7)$ & $35(1.1)$ & 0.039 \\
\hline Nausea & $28(0.8)$ & $4(2.2)$ & $24(0.7)$ & 0.032 \\
\hline Rash & $14(0.4)$ & $2(1.1)$ & $12(0.4)$ & 0.129 \\
\hline Dyspnea & $7(0.2)$ & $2(1.1)$ & $5(0.2)$ & 0.006 \\
\hline Jaundice & $3(0.1)$ & $2(1.1)$ & $1(0.03)$ & $<0.001$ \\
\hline Depression & $3(0.1)$ & $0(0.0)$ & $3(0.1)$ & 0.683 \\
\hline Increase in total bilirubin $^{a}$ & $874(25.1)$ & $103(56.3)$ & $771(23.4)$ & $<0.001$ \\
\hline Grade 1 & $170(4.9)$ & $39(21.3)$ & $131(4.0)$ & $<0.001$ \\
\hline Grade 2 & $663(19.1)$ & $59(32.2)$ & $604(18.0)$ & $<0.001$ \\
\hline Grade 3 & $39(1.1)$ & $5(2.7)$ & $34(1.0)$ & 0.033 \\
\hline Grade 4 & $2(0.1)$ & $0(0.0)$ & $2(0.1)$ & 0.739 \\
\hline Increase in $\mathrm{ALT}^{\mathrm{b}}$ & $55(1.6)$ & $2(1.1)$ & $53(1.6)$ & 0.912 \\
\hline Grade 1 & $28(0.8)$ & $1(0.6)$ & $27(0.8)$ & 0.739 \\
\hline Grade $2^{c}$ & $18(0.5)$ & $1(0.6)$ & $17(0.5)$ & 0.533 \\
\hline Grade $3^{\mathrm{d}}$ & $7(0.2)$ & $0(0.0)$ & $7(0.2)$ & 0.919 \\
\hline Grade $4^{\mathrm{e}}$ & $2(0.1)$ & $0(0.0)$ & $2(0.1)$ & 0.646 \\
\hline Serious adverse events & $21(0.6)$ & $1(0.6)$ & $20(0.6)$ & 0.929 \\
\hline Treatment-related & $1(0.03)$ & $0(0.0)$ & $1(0.03)$ & - \\
\hline Liver & $6(0.2)$ & $0(0.0)$ & $6(0.2)$ & - \\
\hline Digestive & $1(0.03)$ & $0(0.0)$ & $1(0.03)$ & - \\
\hline Cardiovascular & $2(0.06)$ & $0(0.0)$ & $2(0.1)$ & - \\
\hline Endocrine & $1(0.03)$ & $0(0.0)$ & $1(0.03)$ & - \\
\hline Skin & $1(0.03)$ & $1(0.6)$ & $0(0.0)$ & - \\
\hline Infection & $6(0.2)$ & $0(0.0)$ & $6(0.2)$ & - \\
\hline Other cancer & $1(0.03)$ & $0(0.0)$ & $1(0.03)$ & - \\
\hline
\end{tabular}


Table 2 continued

\begin{tabular}{lllll}
\hline & Total $(\boldsymbol{n}=\mathbf{3 4 8 0})$ & + Ribavirin $(\boldsymbol{n}=\mathbf{1 8 3})$ & - Ribavirin $(\boldsymbol{n}=\mathbf{3 2 9 7})$ & $\boldsymbol{P}$ value \\
\hline Other & $2(0.06)$ & $0(0.0)$ & $2(0.06)$ & - \\
\hline
\end{tabular}

$A L T$ alanine aminotransferase, SOF sofosbuvir, $U L N$ upper limit of normal, $V E L$ velpatasvir

aTotal blood bilirubin increased: Grade 1: 1.0 ULN-1.5 $\times$ ULN if baseline was normal; $>1.0-1.5 \times$ baseline if baseline was abnormal; Grade 2: > 1.5-3.0 $\times$ ULN if baseline was normal; > 1.5-3.0 $\times$ baseline if baseline was abnormal; Grade 3: $>3.0-10.0 \times$ ULN if baseline was normal; > 3.0-10.0 $\times$ baseline if baseline was abnormal; Grade 4: > $10.0 \times$ ULN if baseline was normal; $>10.0 \times$ baseline if baseline was abnormal; Normal: BL_Bil(T) $0.1-1.2 \mathrm{mg} / \mathrm{dL}$; abnormal: $\mathrm{BL} \_\mathrm{Bil}(\mathrm{T})>1.2 \mathrm{mg} / \mathrm{dL}$

${ }^{\mathrm{b}}$ ALT increased: Grade 1: $>1.0-3.0 \times$ ULN if baseline was normal; $>1.5-3.0 \times$ baseline if baseline was abnormal; Grade 2: > 3.0-5.0 $\times$ ULN if baseline was normal; > 3.0-5.0 $\times$ baseline if baseline was abnormal; Grade 3: > 5.0-$20.0 \times$ ULN if baseline was normal; > 5.0-20.0 $\times$ baseline if baseline was abnormal; Grade 4: > $20.0 \times$ ULN if baseline was normal; $>20.0 \times$ baseline if baseline was abnormal; Normal: ALT: 4-44 (U/L); abnormal: ALT > $44(\mathrm{U} / \mathrm{L})$

${ }^{\mathrm{c}}$ None were treatment-related; all achieved SVR12

${ }^{\mathrm{d}}$ None were treatment-related; all but one patient achieved SVR12

${ }^{\mathrm{e}}$ None were treatment-related; all achieved SVR12

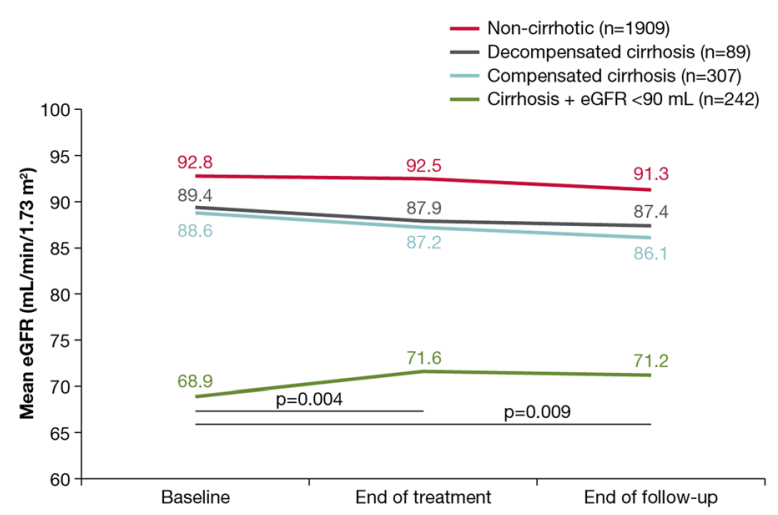

Fig. 2 eGFR at baseline, end of treatment and end of follow-up. Mean eGFR at baseline, end of treatment, and end of follow-up following SOF/VEL therapy stratified by cirrhosis stage. $e G F R$ estimated glomerular filtration rate

genotype 3. Of the 97 patients with genotype 3 infection, approximately $97 \%$ were cured. Of note, 91 of these patients were treated without the addition of ribavirin. Until recently there had been some caution around using SOF/VEL without ribavirin in patients with genotype 3 and cirrhosis. However, the high rates achieved without ribavirin in this difficult-to-cure cohort are not only in line with the phase 3 ASTRAL-3 and POLARIS-3 trials (cure rates between 93\% and $98 \%$ in patients with genotype $3[6,22]$ ) but also exceed the SVR12 rates observed in the
Asian phase 3 trial (76\%) [11]. However, the Asian phase 3 trial included a high proportion of patients with genotype $3 \mathrm{~b}$ infection, who are considered more resistant to treatment with SOF/VEL or glecaprevir/pibrentasvir (GLE/PIB) [23], but account for only approximately $10 \%$ of the genotype 3 population $[16,24]$. The results from this cohort study are also in line with a recent large-scale real-world study across Europe and North America which reported SVR12 rates of $98.3 \%$ among 1677 patients with genotype 3 $\mathrm{HCV}$ treated without ribavirin [8]. The high response rates seen with SOF/VEL in genotype 3 patients, even those with cirrhosis, have been reflected recently in the changing guidelines [2]. This patient group can now be considered for a simplified regimen and initiation of pangenotypic therapy without the need for genotyping or assessment of fibrosis.

Although the mean number of outpatient visits from initiating treatment to SVR12 assessment in this TACR cohort was relatively high, this is due to advice from the Taiwan Health Insurance Administration. Patients, especially those with cirrhosis, are advised to visit every 2 weeks for $4-8$ weeks during DAA treatment, followed by every 4 weeks posttreatment for reimbursement purposes. However, following recently published data demonstrating high SVR12 rates achieved with 
a minimal monitoring treatment schedule, it could be argued that this may not be required [25].

HCV populations have more comorbidities that may aggravate the progression of fibrosis, such as obesity and insulin resistance and coinfection with HIV or HBV. The development of hepatic and extrahepatic diseases, such as circulatory disease and renal disease, can lead to increased mortality in patients with chronic HCV [26]. In Taiwan, the most common comorbidities are diseases of the digestive system $(40.1 \%)$, circulatory system $(38.7 \%)$, and endocrine/nutritional/metabolic diseases (35.2\%). These diverse comorbidities in the Taiwanese population are reflected in this current cohort and it is encouraging that high SVR12 rates were achieved with SOF/VEL in persons who inject drugs (99.5\%), patients with active HCC (98.5\%), and those coinfected with HBV $(98.8 \%)$ or HIV (96.9\%). This suggests that SOF/VEL was well tolerated in patients with comorbidities and these rates of cure match or exceed those of previous clinical and real-world studies [27-29].

Currently, there is a large gap in data on the effectiveness and safety of SOF/VEL in patients with decompensated cirrhosis in Taiwan. As the use of PI-based regimens is contraindicated in patients with decompensated cirrhosis, such data are essential for guiding patients and physicians in treatment decision-making and advising healthcare policies on treatment coverage. To the best of our knowledge, this is the largest cohort study on the efficacy and safety of SOF/VEL treatment in patients with decompensated cirrhosis in Taiwan. The high SVR12 rates achieved in this patient group in this study (100\% of 114 patients) demonstrate the high effectiveness and value of a PI-free regimen, such as SOF/VEL, in this patient group.

In patients who did not achieve SVR12, HCV RNA viral load over $6,000,000 \mathrm{IU} / \mathrm{mL}$ and $\mathrm{HCV}$ genotype 3 infection were the factors found to be significantly associated with the risk of not achieving SVR12. However, as only three patients with genotype 3 did not achieve SVR in the TACR cohort, and all patients with genotype 3 and high viral load achieved cure, this statistically significant difference is unlikely to have a clinical impact, especially given that the majority of the chronic HCV population in Taiwan is infected with genotype 1 or 2 (as also reflected in this cohort). Despite having a very low cutoff value for drug adherence (the SIMPLIFY study quantified low adherence as below $90 \%$ [30]), the majority of patients (5/9) with adherence below this $60 \%$ threshold achieved SVR12 with SOF/VEL. However, adherence is important to ensure treatment effectiveness.

As the metabolite of SOF is renally cleared, SOF-based regimens were previously not recommended in patients with severely impaired renal function (CKD stage 4 or 5, eGFR $<30 \mathrm{~mL} / \mathrm{min} / 1.73 \mathrm{~m}^{2}$ ). However, despite elevated plasma levels of sofosbuvir and its metabolite GS-331007 in patients with renal impairment, increasing evidence supports the safety and effectiveness of SOF-based regimens in this patient population [31, 32], including those with liver cirrhosis [32] and uremic patients on dialysis [33]. In November 2019, the US Food and Drug Administration (FDA) amended the package inserts for SOF-based regimens to allow use in patients with renal disease, including those with an eGFR $<30 \mathrm{~mL} /$ $\min / 1.73 \mathrm{~m}^{2}$ and those on dialysis, and the Taiwan consensus statement on the management of hepatitis C recommends SOF/VEL as one of the treatment options for patients with stage 4 or 5 CKD [17]. This is supported by data from this cohort that demonstrated a lack of fluctuation in eGFR levels across some CKD stages and, notably, all patients with CKD stage 4 achieved SVR12. In patients with eGFR $<90 \mathrm{~mL} / \mathrm{min} / 1.73 \mathrm{~m}^{2}$ and cirrhosis, eGFR increased significantly, showing that not only could renal function deterioration be halted but it could also be improved in some patient groups typically seen as "difficult to cure".

The majority of patients tolerated treatment well with only $10 \%$ of patients experiencing adverse events and $0.6 \%$ experiencing serious adverse events, the majority of which were experienced in the ribavirin-added group. Only three patients discontinued treatment as a result of adverse events and these were not considered to be related to SOF/VEL therapy. It should be noted that the adverse events observed could be due to ribavirin or the 
severity of disease. Although, with the high SVR12 rates achieved irrespective of ribavirin, it raises the question whether a simplified therapy without ribavirin would be more beneficial. This has been highlighted by a recent phase 3 trial from Japan that demonstrated SOF/VEL without ribavirin was highly effective and well tolerated in patients with decompensated cirrhosis. The study found the addition of ribavirin did not improve efficacy, but increased toxicity [34].

There were limitations identified in this cohort study. First, this was a retrospectiveprospective study, where patients treated with SOF/VEL before February 2021 were eligible for inclusion. However, the data analyzed were obtained prospectively in accordance with the regulations of Taiwan's Health Insurance Administration for DAA therapy. Second, as a result of limited numbers of certain types of patients, such as patients with HCV genotypes 4 and 5, the treatment outcomes for those patients may be lacking. Despite these limitations, the TACR includes data from patients treated at a range of settings across Taiwan, including medical centers, regional hospitals, and local clinics. Furthermore, as no specific exclusion criteria were applied to the study population, this is a true reflection of the realworld management of patients with chronic hepatitis $C$ in this country.

\section{CONCLUSION}

A simple, pangenotypic, protease inhibitor-free treatment that results in high SVR12 rates with and without ribavirin, regardless of genotype, cirrhosis, severity of liver disease, and comorbidities is of value in Taiwan and across Asia. The confirmation of the high SVR12 rates with SOF/VEL with and without ribavirin supports use of this regimen in a more diverse population, including those with decompensated cirrhosis and CKD.

\section{ACKNOWLEDGEMENTS}

Funding. The study was partly supported by grants from Kaohsiung Medical University (MOST 109-2314-B-037-044, KMU-K1110002, MOST 108-2314-B-037-066-MY3, KMUTC108B06 [Center for Liquid Biopsy], KMUTC108B07 \& KMU-DK109002 [Cohort Research Center], 105KMUOR08 [Center for Cancer Research KMU Global Networking Talent Plan Grant], and KMU-TC109B05 [Center for Liquid Biopsy and Cohort Research]) and Kaohsiung Medical University Hospital (KMUH109-9R06, KMUH109-9R05, MOHW109-TDU-B-212114006 and KMUH-IIT-109-2-05). The journal's Rapid Service Fee is funded by Gilead Sciences Hong Kong Ltd.

Medical Writing Assistance. Medical writing assistance was provided by Ridda Jabbar and Julia Heagerty from Elements Communications Ltd, United Kingdom, funded by Gilead Sciences Hong Kong Ltd. Gilead had no control over the final content of this manuscript.

Authorship. All named authors meet the International Committee of Medical Journal Editors (ICMJE) criteria for authorship for this article, take responsibility for the integrity of the work as a whole, and have given their approval for this version to be published.

Author Contributions. Ming-Lung Yu, HanChieh Lin and Pin-Nan Cheng were responsible for the study conception and design, all authors contributed to the study design and data collection, and analyses were performed by MingLung Yu, Han-Chieh Lin and Pin-Nan Cheng. All authors reviewed early drafts of the manuscript and read and approved the final version for publication.

Prior Presentation. Part of this work was presented at the International Symposium on Viral Hepatitis and Liver Disease Global Hepatitis Summit held between June 17 and June 20, 2021, in Taipei, Taiwan. \#P204. 
Disclosures. Pin-Nan Cheng: Advisory board for AbbVie, Gilead Sciences, and Merck Sharp \& Dohme. Speaker for Abbott, AbbVie, Gilead Sciences, and Merck Sharp \& Dohme. Research grants from Gilead Sciences and Merck Sharp \& Dohme. Jia-Horng Kao: Speaker, consultant and/or advisory board member for AbbVie, Arbutus Biopharma, Bristol Myers Squibb, Fujirebio, Gilead Sciences, Johnson \& Johnson, MSD, Polaris, Sysmex and Roche. Ming-Lung Yu: Research support (grant) from Abbott, BMS, Gilead Sciences and Merck Sharp \& Dohme. Consultant of AbbVie, Abbott, BMS, Gilead Sciences, Merck Sharp \& Dohme, and Roche Diagnostics. Speaker for AbbVie, Abbott, BMS, Gilead Sciences, IPSEN, Merck Sharp \& Dohme and Roche. Chung-Feng Huang: Speaker for AbbVie, BMS, Gilead Sciences, Merck Sharp \& Dohme, and Roche. Chen-Hua Liu: Advisory board for AbbVie, Gilead Sciences, and Merck Sharp \& Dohme. Speaker for Abbott, AbbVie, Gilead Sciences, and Merck Sharp \& Dohme. Research grants from AbbVie, Gilead Sciences, and Merck Sharp \& Dohme. All other authors (Lein-Ray Mo, Chun-Ting Chen, Chi-Yi Chen, Hsing-Tao Kuo, Ching-Chu Lo, Kuo-Chih Tseng, Yi-Hsiang Huang, Chi-Ming Tai, ChengYuan Peng, Ming-Jong Bair, Chien-Hung Chen, Ming-Lun Yeh, Chih-Lang Lin, Chun-Yen Lin, Pei-Lun Lee, Lee-Won Chong, Chao-Hung Hung, Te Sheng Chang, Jee-Fu Huang, ChiChieh Yang, Jui-Ting Hu, Chih-Wen Lin, ChiaChi Wang, Wei-Wen Su, Tsai-Yuan Hsieh, ChihLin Lin, Wei-Lun Tsai, Tzong-Hsi Lee, Guei-Ying Chen, Szu-Jen Wang, Chun-Chao Chang, Sheng-Shun Yang, Wen-Chih Wu, Chia-Sheng Huang, Kwok-Hsiung Chou, Chien-Neng Kao, Pei-Chien Tsai, Mei-Hsuan Lee, Chien-Yu Cheng, Ming-Chang Tsai, Chun-Jen Liu, ChiaYen Dai, Han-Chieh Lin, Wan-Long Chuang): Nothing to disclose.

Compliance with Ethics Guidelines. Ethics approval was granted by Kaohsiung Medical University Chung-Ho Memorial Hospital (IRB number KMUHIRB-F(I)-20170053).

Data Availability. The datasets generated and analyzed during the current study are available from the corresponding author on reasonable request.

Contributors. The authors would also like to thank the Taiwan Association for the Study of the Liver (TASL), the TASL Foundation, and Taiwan Liver Research Foundation for grant support and the TACR study group for data collection. We also thank the Center for Medical Informatics and Statistics of Kaohsiung Medical University for providing administrative and funding support.

Open Access. This article is licensed under a Creative Commons Attribution-NonCommercial 4.0 International License, which permits any non-commercial use, sharing, adaptation, distribution and reproduction in any medium or format, as long as you give appropriate credit to the original author(s) and the source, provide a link to the Creative Commons licence, and indicate if changes were made. The images or other third party material in this article are included in the article's Creative Commons licence, unless indicated otherwise in a credit line to the material. If material is not included in the article's Creative Commons licence and your intended use is not permitted by statutory regulation or exceeds the permitted use, you will need to obtain permission directly from the copyright holder. To view a copy of this licence, visit http://creativecommons.org/licenses/bync/4.0/.

\section{REFERENCES}

1. Polaris Observatory HCV Collaborators. Global prevalence and genotype distribution of hepatitis C virus infection in 2015: a modelling study. Lancet Gastroenterol Hepatol. 2017;2:161-71.

2. European Association for the Study of the Liver. EASL recommendations on treatment of hepatitis C: final update of the series. J Hepatol. 2020;73: 1170-218.

3. Backus LI, Boothroyd DB, Phillips BR, Belperio P, Halloran J, Mole LA. A sustained virologic response reduces risk of all-cause mortality in patients with hepatitis C. Clin Gastroenterol Hepatol. 2011;9: 509-16. 
4. van der Meer A, Veldt B, Feld JJ, et al. Association between sustained virological response and allcause mortality among patients with chronic hepatitis $\mathrm{C}$ and advanced hepatic fibrosis. JAMA. 2012;308:2584-93.

5. Feld J, Jacobson IM, Hézode C, et al. Sofosbuvir and velpatasvir for HCV genotype 1, 2, 4, 5, and 6 infection. N Engl J Med. 2015;373:2599-607.

6. Foster G, Afdhal N, Roberts S, et al. Sofosbuvir and velpatasvir for HCV genotype 2 and 3 infection. N Engl J Med. 2015;373:2608-17.

7. Curry M, O'Leary J, Bzowej N, et al. Sofosbuvir and velpatasvir for $\mathrm{HCV}$ in patients with decompensated cirrhosis. N Engl J Med. 2015;373:2618-28.

8. Mangia A, Milligan S, Khalili M, et al. Global realworld evidence of sofosbuvir/velpatasvir as simple, effective HCV treatment: analysis of 5552 patients from 12 cohorts. Liver Int. 2020;40:1841-52.

9. Belperio P, Shahoumian T, Loomis T, Mole L, Backus L. Real-world effectiveness of daclatasvir plus sofosbuvir and velpatasvir/sofosbuvir in hepatitis C genotype 2 and 3. J Hepatol. 2019;70:15-23.

10. Buggisch P, Wursthorn K, Stehr A, et al. Real-world effectiveness and safety of sofosbuvir/velpatasvir and ledipasvir/sofosbuvir hepatitis $C$ treatment in a single centre in Germany. PLoS ONE. 2019;14: e0214795.

11. Wei L, Lim SG, Xie Q, et al. Sofosbuvir-velpatasvir for treatment of chronic hepatitis $C$ virus infection in Asia: a single-arm, open-label, phase 3 trial. Lancet Gastroenterol Hepatol. 2019;4:127-34.

12. Yang J-F, Lin C-I, Huang J-F, et al. Viral hepatitis infections in southern Taiwan: a multicenter community-based study. Kaohsiung J Med Sci. 2010;26: 461-9.

13. Yu M-L, Yeh M-L, Tsai P-C, et al. Huge gap between clinical efficacy and community effectiveness in the treatment of chronic hepatitis C: a nationwide survey in Taiwan. Medicine. 2015;94:e690.

14. Bennet $\mathrm{H}$, Waser $\mathrm{N}$, Johnston $\mathrm{K}$, et al. A review of the burden of hepatitis $\mathrm{C}$ virus infection in China, Japan, South Korea and Taiwan. Hepatol Int. 2015;9:378-90.

15. Yu M-L, Chuang W-L. Treatment of chronic hepatitis C in Asia: when East meets West. Hepatology. 2009;24:336-45.

16. Lu M-Y, Chen C-T, Shih Y-L, et al. Changing epidemiology and viral interplay of hepatitis B, C and $\mathrm{D}$ among injecting drug user-dominant prisoners in Taiwan. Sci Rep. 2021;11:8554.
17. Yu M-L, Chen PJ, Dai CY, et al. 2020 Taiwan consensus statement on the management of hepatitis C: Part (II) special populations. J Formos Med Assoc. 2020;119:1135-57.

18. Chen CY, Huang CF, Cheng PN, et al. Factors associated with treatment failure of direct-acting antivirals for chronic hepatitis C: a real-world nationwide hepatitis $C$ virus registry programme in Taiwan. Liver Int. 2021;41:1265-77.

19. Liu CH, Huang YJ, Yang SS, et al. Generic sofosbuvir-based interferon-free direct acting antiviral agents for patients with chronic hepatitis $C$ virus infection: a real-world multicenter observational study. Sci Rep. 2018;8:13699.

20. Kowdley KV, Gordon SC, Reddy KR, et al. Ledipasvir and sofosbuvir for 8 or 12 weeks for chronic HCV without cirrhosis. N Engl J Med. 2014;370:1879-88.

21. Liu CH, Chen PY, Chen JJ, et al. Sofosbuvir/velpatasvir for patients with chronic hepatitis $C$ virus infection and compensated liver disease: real-world data in Taiwan. Hepatol Int. 2021;15:338-49.

22. Jacobson IM, Lawitz E, Gane EJ, et al. Efficacy of 8 weeks of sofosbuvir, velpatasvir and voxilaprevir in patients with chronic HCV infection: two phase 3 randomized trials. Gastroenterology. 2017;153: 113-22.

23. Smith D, Magri A, Bonsall D, et al. Resistance analysis of genotype 3 hepatitis $C$ virus indicates subtypes inherently resistant to nonstructural protein 5A inhibitors. Hepatology. 2019;69:1861-71.

24. Hsieh M-H, Tsai J-J, Hsieh M-Y, et al. Hepatitis C virus infection among injection drug users with and without human immunodeficiency virus co-infection. PLoS ONE. 2014;9(4): e94791.

25. Solomon S, Wagner-Cardoso S, Smeaton L, et al. The "keep it simple and safe" approach to HCV treatment: primary outcomes from the ACTG A5360 (MINMON) study. Presented at the AASLD 2020 Liver Meeting Digital Experience; November 11-16, 2020. Abstract LO7.

26. Yu M-L, Chen PJ, Dai CY, et al. 2020 Taiwan consensus statement on the management of hepatitis C: part (I) general population. J Formos Med Assoc. 2020;119:1019-40.

27. Wyles D, Bräu N, Kottilil S, et al. Sofosbuvir and velpatasvir for the treatment of hepatitis $C$ virus in patients coinfected with human immunodeficiency virus type 1: an open-label, phase 3 study. Clin Infect Dis. 2017;65:6-12.

28. Yeh M-L, Huang C-F, Huang C-I, et al. Hepatitis B-related outcomes following direct-acting antiviral 
therapy in Taiwanese patients with chronic HBV/ HCV co-infection. J Hepatol. 2020;71:62-71.

29. Grebely J, Dalgard O, Conway B, et al. Sofosbuvir and velpatasvir for hepatitis $C$ virus infection in people with recent injection drug use (SIMPLIFY): an open-label, single-arm, phase 4 , multicentre trial. Lancet Gastroenterol Hepatol. 2018;3:153-61.

30. Cunningham EB, Amin J, Feld J, et al. Adherence to sofosbuvir and velpatasvir among people with chronic HCV infection and recent injection drug use: the SIMPLIFY study. Int J Drug Policy. 2018;62: 14-23.

31. Eletreby R, El-Serafy M, Anees M, et al. Sofosbuvircontaining regimens are safe and effective in the treatment of HCV patients with moderate to severe renal impairment. Liver Int. 2019;40:797-805.

32. Liu C-H, Chen C-H, Su W-W, et al. Sofosbuvir/velpatasvir with or without low-dose ribavirin for patients with chronic hepatitis $C$ virus infection and severe renal impairment. Gut. 2022;71:176-84.

33. Yu M-L, Huang C-F, Wei Y-J, et al. Establishment of an outreach, grouping healthcare system to achieve microelimination of $\mathrm{HCV}$ for uremic patients in haemodialysis centres (ERASE-C). Gut. 2021;70: 2349-58.

34. Takaoka Y, Miura K, Morimoto N, et al. Real-world efficacy and safety of 12-week sofosbuvir/velpatasvir treatment for patients with decompensated liver cirrhosis caused by hepatitis $C$ virus infection. Hepatol Res. 2022;71:176-84.

\section{Publisher's Note}

Springer Nature remains neutral with regard to jurisdictional claims in published maps and institutional affiliations. 